\title{
Comprehensive geriatric assessment is an essential tool to support treatment decisions in elderly patients with diffuse large B-cell lymphoma: a prospective multicenter evaluation in 173 patients by the Lymphoma Italian Foundation (FIL)
}

\begin{abstract}
Alessandra Tucci, Maurizio Martelli, Luigi Rigacci, Paola Riccomagno, Maria Giuseppina Cabras, Flavia Salvi, Caterina Stelitano, Alberto Fabbri, Sergio Storti, Stefano Fogazzi, Salvatrice Mancuso, Maura Brugiatelli, Angelo Fama, Paolo Paesano, Benedetta Puccini, Chiara Bottelli, Daniela Dalceggio, Francesco Bertagna, Giuseppe Rossi, Michele Spina \& for the Italian Lymphoma Foundation (FIL)
\end{abstract}

To cite this article: Alessandra Tucci, Maurizio Martelli, Luigi Rigacci, Paola Riccomagno, Maria Giuseppina Cabras, Flavia Salvi, Caterina Stelitano, Alberto Fabbri, Sergio Storti, Stefano Fogazzi, Salvatrice Mancuso, Maura Brugiatelli, Angelo Fama, Paolo Paesano, Benedetta Puccini, Chiara Bottelli, Daniela Dalceggio, Francesco Bertagna, Giuseppe Rossi, Michele Spina \& for the Italian Lymphoma Foundation (FIL) (2015) Comprehensive geriatric assessment is an essential tool to support treatment decisions in elderly patients with diffuse large B-cell lymphoma: a prospective multicenter evaluation in 173 patients by the Lymphoma Italian Foundation (FIL), Leukemia \& Lymphoma, 56:4, 921-926, DOI: 10.3109/10428194.2014.953142

To link to this article: http://dx.doi.org/10.3109/10428194.2014.953142

View supplementary material $₫$

Accepted author version posted online: 11 Aug 2014. Published online: 09 Oct 2014.

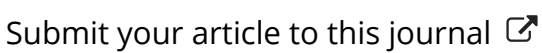

ЦIl Article views: 686 
Citing articles: 15 View citing articles ๘

Full Terms \& Conditions of access and use can be found at http://www.tandfonline.com/action/journallnformation?journalCode=ilal20 


\title{
Comprehensive geriatric assessment is an essential tool to support treatment decisions in elderly patients with diffuse large B-cell lymphoma: a prospective multicenter evaluation in 173 patients by the Lymphoma Italian Foundation (FIL)
}

\author{
Alessandra Tucci ${ }^{1}$, Maurizio Martelli ${ }^{2}$, Luigi Rigacci ${ }^{3}$, Paola Riccomagno ${ }^{4}$, Maria Giuseppina Cabras 5 , \\ Flavia Salvi ${ }^{6}$, Caterina Stelitano ${ }^{7}$, Alberto Fabbri ${ }^{8}$, Sergio Storti ${ }^{9}$, Stefano Fogazzi ${ }^{1}$, Salvatrice Mancuso ${ }^{10}$, \\ Maura Brugiatelli ${ }^{11}$, Angelo Fama2 ${ }^{2}$, Paolo Paesano2 ${ }^{2}$ Benedetta Puccini ${ }^{3}$, Chiara Bottelli ${ }^{1}$, Daniela Dalceggio ${ }^{1}$, \\ Francesco Bertagna ${ }^{12}$, Giuseppe Rossi ${ }^{1} \&$ Michele Spina ${ }^{13}$; for the Italian Lymphoma Foundation (FIL) \\ ${ }^{1}$ Department of Hematology, Spedali Civili, Brescia, Italy, ${ }^{2}$ Department of Cellular Biotechnologies and Hematology, \\ University Sapienza Roma, Italy, ${ }^{3}$ Department of Hematology, "Careggi" University Hospital, Firenze, Italy, ${ }^{4}$ Città della salute \\ e della Scienza Hospital, Torino, Italy, ${ }^{5}$ Department of Hematology, Oncologic Hospital "Armando Businco", Cagliari, Italy, \\ ${ }^{6}$ Hematology Division, SS Antonio e Biagio Hospital, Alessandria, Italy, ${ }^{7}$ Hematology Division, "Bianchi, Melacrino, Morelli" \\ Hospital, Reggio Calabria, Italy, ${ }^{8}$ Division of Hematology, University Hospital of Siena, Italy, ${ }^{9}$ Onco-Hematology Division, \\ Catholic University - "Giovanni Paolo II" Foundation, Campobasso, Italy, ${ }^{10}$ Unit of Hematology and Transplantation, \\ "Paolo Giaccone" University Hospital, Palermo, Italy, "Department of Hematology, "Papardo" Hospital, Messina, Italy, \\ ${ }^{12}$ Nuclear Medicine, Brescia University, Italy and ${ }^{13}$ Department of Medical Oncology, National Cancer Institute, Aviano, Italy
}

\begin{abstract}
We performed a multicenter study to validate the concept that a simple comprehensive geriatric assessment (CGA) can identify elderly, non-fit patients with diffuse large B-cell lymphoma (DLBCL) in whom curative treatment is not better then palliation, and to analyze potential benefits of treatment modulation after further subdividing the non-fit category by CGA criteria. One hundred and seventy-three patients aged $>69$ treated with curative or palliative intent by clinical judgement only were grouped according to CGA into fit (46\%), unfit (16\%) and frail $(38 \%)$ categories. Two-year overall survival (OS) was significantly better in fit than in non-fit patients ( $84 \%$ vs. $47 \% ; p<0.0001)$. Survival in unfit and frail patients was not significantly different. Curative treatment slightly improved 2 -year OS in unfit $(75 \%$ vs. $45 \%$ ) but not in frail patients ( $44 \%$ vs. $39 \%$ ). CGA was confirmed as very efficient in identifying elderly patients with DLBCL who can benefit from a curative approach. Further efforts are needed to better tailor therapies in non-fit patients.
\end{abstract}

Keywords: Lymphoma and Hodgkin disease, chemotherapeutic approaches, immunotherapy

\section{Introduction}

In the last few years, comprehensive geriatric assessment (CGA) has been recognized as an essential tool for clinicians who manage elderly people affected by aggressive diseases [1].
The definition of elderly is still a matter of debate in oncology. In patients with lymphoma, the age limit more frequently used to discriminate between younger and older patients is 65 years [2].

It is no longer acceptable to exclude patients from intensive treatment with curative intent only on the basis of chronological age. Aging is highly individualized in terms of life expectancy, functional reserve and social support, and treatment plans need to account for this diversity that can be better evaluated by a multidimensional approach [3].

Diffuse large B-cell lymphoma (DLBCL) represents a typical example of neoplastic disease that can be cured with an aggressive program. The majority of patients with DLBCL are over the age of 60 years, and the steady improvement of life expectancy will probably further increase their number in the future [2]. Therefore, trying to improve the survival of this category of patients represents one of the major challenges for hematologists. CGA proved to be an effective method to identify elderly patients with DLBCL able to tolerate intensive treatment with curative intent, achieving an outcome similar to that of younger patients [4]. However, about one-half of elderly patients cannot benefit from a curative approach, and their prognosis is still very poor, independent of treatment received.

Although broad agreement exists among oncologists on the different aspects of geriatric assessment and the instruments to use for evaluating the performance of elderly

Correspondence: Alessandra Tucci, Department of Hematology, Spedali Civili, Brescia, Italy. Tel: 0039303995438. Fax: 0039303700852. E-mail: alessandra. tucci@spedalicivili.brescia.it

There is an accompanying commentary that discusses this paper. Please refer to the issue Table of Contents.

Received 3 November 2013; revised 9 July 2014; accepted 3 August 2014 
Table I. Definition of three geriatric risk categories according to age, comorbidities and functional abilities of daily living.

\begin{tabular}{|c|c|c|c|}
\hline & \multicolumn{3}{|c|}{ CGA category } \\
\hline & Fit & Unfit & Frail \\
\hline ADL & 6 & $5^{*}$ & $\leq 4^{*}$ \\
\hline IADL & 8 & $6-7^{*}$ & $\leq 5^{*}$ \\
\hline CIRS-G & $\begin{array}{l}\text { No comorbidity score } 3-4 \text { and } \\
<5 \text { comorbidities score } 2\end{array}$ & $\begin{array}{l}\text { No comorbidity score } 3-4 \\
\text { and 5-8 comorbidities } \\
\text { score } 2\end{array}$ & $\begin{array}{l}\geq 1 \text { Comorbidity score } 3-4 \\
\quad \text { or }>8 \text { comorbidities } \\
\text { score } 2\end{array}$ \\
\hline Age & & $\geq 80$ fit & $\geq 80$ unfit \\
\hline
\end{tabular}

ADL, activity of daily living; IADL, instrumental activity of daily living; CIRS-G, Cumulative Illness Rating Score for Geriatrics; CGA, comprehensive geriatric assessment.

*Number of residual functions.

people, the categories of elderly patients that need to be identified are still debated, and interpretation of the results of CGA tests has not yet been standardized. In particular, since new and very effective treatments with a better tolerability profile such as anti-CD20 monoclonal antibodies have become available, the attempt to define non-fit patients according to the extent of their limitations could help clinicians to better tailor treatment in an attempt to improve the efficacy of therapies in this patient category.

Therefore the Italian Lymphoma Foundation (FIL) launched a multicenter prospective study to verify our previous results in a broader population of patients with DLBCL with the same clinical characteristics, and to assess the potential usefulness of a CGA that further divides non-fit patients into two different levels according to the severity of their unfitness.

\section{Patients and methods}

All consecutive patients with DLBCL aged more than 69 years seen in 13 hematology departments of the FIL were recorded during 1 year. CGA was performed during staging procedures, after written informed consent, through application of the following instruments: (1) age $>80$ years; (2) comorbidity score according to the Cumulative Illness Rating Score for Geriatrics (CIRS-G) [5] and evaluated in all organs/systems as follows: no problem -0 , mild problem (may require treatment) - 1, moderate disability or morbidity (treatment required) -2 , severe, constant, significant disability/"uncontrollable" chronic problems - 3, extremely severe disability, immediate treatment required/end organ failure/severe impairment in function - 4; (3) activity of daily living (ADL), that is, loss in any activity, including bathing, dressing, toileting, transferring, feeding and continence [6];
(4) instrumental activity of daily living (IADL), an indirect evaluation of functional abilities necessary for independent living through caregiver interview [7]. Patients were classified in the category of "fit" patients if they had all of the following conditions: age $<80$, no limitations in ADL and IADL scores: ADL score 6 and IADL score 8, CIRS-G: no grade 3-4 comorbidities (hematological comorbidities were not investigated) and fewer than five grade 2 comorbidities. Patients were classified as "unfit" if they had all of the following conditions: age $>79$, no limitations in ADL and IADL scores: ADL score 6 and IADL score 8, CIRS-G: no grade 3-4 comorbidities and fewer than five grade 2 comorbidities. In addition, patients aged $<80$ with an ADL score of 5 and/ or IADL score of 6-7, and/or CIRS-G: no grade 3-4 comorbidities and 5-8 grade 2 comorbidities were also classified as "unfit." All other patients who did not meet the criteria for fit and unfit patients were classified as "frail" [8] (Table I). The decision to treat a patient and the choice of type and intensity of treatment were always left to the clinical judgement of the attending physician, according to the policy of the institution. Treatment with curative intent was defined as the use of a combination of an anthracycline-based chemotherapy (either liposomal or standard formulation), consisting of CHOP (cyclophosphamide, doxorubicin, vincristine, prednisone) or CHOP-like regimens with rituximab, delivered at a relative dose intensity greater than $70 \%$ of the full dose as intent-to-treat. Patients considered unable to tolerate such treatment received other treatments, including radiation therapy only, low-dose chemotherapy without anthracyclines (cyclophosphamide, vincristine, prednisone [COP], low-dose COP), rituximab as a single agent, corticosteroids alone, oral monochemotherapy or anthracycline-based cycles at a relative dose intensity less than $70 \%$, which were all defined as palliative therapy.

Table II. Characteristics of patients classified according to CGA*.

\begin{tabular}{|c|c|c|c|c|c|}
\hline CGA category & All & Fit & Unfit & Frail & $p$-Value \\
\hline No. of evaluable patients (\%) & 173 & $79(46 \%)$ & $28(16 \%)$ & $66(38 \%)$ & \\
\hline $\mathrm{M} / \mathrm{F}$ & $91 / 82$ & $52 / 27$ & $13 / 15$ & $26 / 40$ & \\
\hline Median age & 77 & 74 & 79 & 81 & $<0.0001$ \\
\hline Ann Arbor stage III-IV $(\%)^{\dagger}$ & 57 & 57 & 58 & 58 & NS \\
\hline B symptoms $(\%)^{\ddagger}$ & 32 & 25 & 24 & 37 & NS \\
\hline IPI risk class intermediate-high/high $(\%)^{\S}$ & 43 & 41 & 44 & 54 & NS \\
\hline
\end{tabular}

CGA, comprehensive geriatric assessment; IPI, International Prognostic Index; NS, not significant.

*Data were available for $100 \%$ of patients in analysis except:

†Stage: missing data in $3 \%$.

${ }^{\ddagger}$ B symptoms: missing data in $30 \%$.

SIPI: missing data in $6 \%$. 


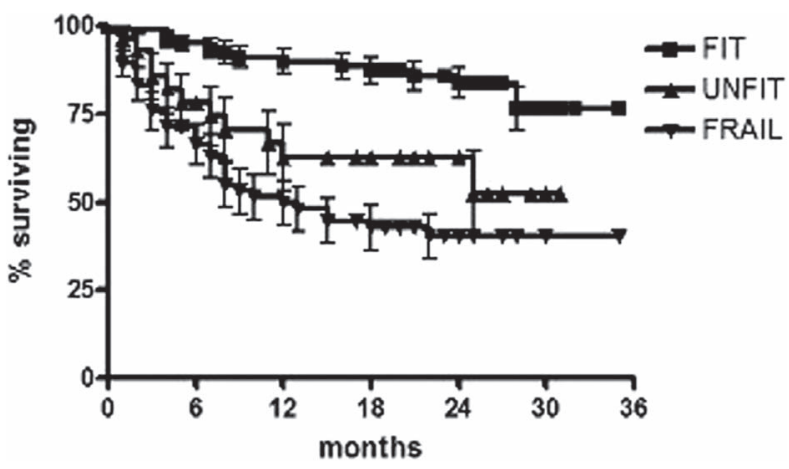

Figure 1. Actuarial overall survival curves of elderly patients with DLBCL classified as "fit," "unfit" and "frail" according to CGA, independent of treatment received.

The purpose of the study was to evaluate the outcome of consecutive patients considering both the intensity of treatment received and the results of CGA assessment.

Statistical analyses were performed using Prism software (GraphPad Software, La Jolla, CA). The overall survival (OS) time was computed from the initiation of therapy to the last visit that the patient was known to be alive or death from any cause, and was evaluated according to the Kaplan-Meier method [9]. The characteristics of the subgroups of patients divided according to the type of treatment received and CGA categorization were compared using the Fisher exact test, Student $t$-test and Mann-Whitney statistics, as appropriate. Log-rank analysis was used to compare actuarial survival curves [10]. The multivariate analysis of survival was performed using the Cox proportional hazard ratio (HR) [11] and $95 \%$ confidence interval (CI), taking into account all variables that had been shown to be significantly associated with survival in the univariate analysis.

\section{Results}

From September 2009 to August 2010, 177 patients aged more than 69 and affected by DLBCL were consecutively registered, and 173 of them had fully evaluable data to be considered for the present study. According to CGA, 79 (46\%) patients were classified as "fit," 28 (16\%) as "unfit" and 66 (38\%) as "frail." Two fit, one unfit and one frail patient were lost to follow-up. Among unfit patients, 50\% were aged more than 79 without any other limitation, $15 \%$ had one or two limitations in IADL only, $23 \%$ in IADL and ADL, and $12 \%$ in IADL, with more than four comorbidities of grade 2 . Among frail patients, $13 \%$ were aged more than 79 and unfit, $15 \%$ had one or more comorbidities of grade 3, two patients had serious limitation only in IADL, while all other patients had limitations in two or more assessment scales. The main demographic and clinical characteristics of this geriatric population are reported in Table II. Fit patients were significantly younger compared with unfit and frail patients $(p<0.0001)$, while no significant difference concerning stage, B symptoms and International Prognostic Index (IPI) was observed. All but five fit patients (94\%) received a curative treatment: one died before receiving any treatment, three patients with stage I disease received only radiation therapy and one patient with stage II disease received radiation and immunochemotherapy without antracycline because of previous treatment for breast cancer. With a median follow-up of 24 months, their 2-year OS was significantly better than the survival of non-fit patients ( $84 \%$ vs. $47 \%$ ) $(p<0.0001)$. Among unfit and frail patients the 2-year survival was $63 \%$ vs. $40 \%$, respectively ( $p=0.13$ ) (Figure 1 ).

On the basis of clinical judgement, 17 unfit patients (61\%) and 18 frail patients $(27 \%)$ received full-dose therapy with curative intent, and the remaining patients received palliation. Clinical characteristics of patients subdivided according to treatment actually received are shown in Table III and Supplementary Figure 1 to be found online at http://informahealthcare.com/doi/abs/10.3109/10428194. 2014.953142. Patients treated with curative intent were significantly younger than patients receiving palliation $(p=0.001)$ and had a more advanced stage $(p=0.02)$, while no other significant differences were recorded.

Considering patients treated with curative intent overall, the survival of fit patients was significantly better than the survival of non-fit patients ( $88 \%$ vs. $56 \%)(p=0.0001)$.

Within the single CGA categories, the 2 -year OS of patients treated with curative or palliative intent was $88 \%$ vs. $25 \%$ $(p=0.0001)$ in fit, $75 \%$ vs. $45 \%$ ( $p=0.32$ ) in unfit and $44 \%$ vs. $39 \%(p=0.75)$ in frail patients, respectively (Figure 2).

The rate of non-hematologic toxicity of grade $>2$ was not significantly different among patients treated, respectively, with curative or palliative intent ( $45 \%$ vs. $38 \%)$ ( $p=0.3$ ). Lymphoma was the main cause of death in both treatment groups.

At univariate analysis, age, IPI, CGA, ADL, IADL, CIRS-G, treatment approach (curative vs. palliative) and treatment dose-intensity (cut-off $70 \%$ ) were significantly associated

Table III. Characteristics of unfit and frail patients subdivided according to type of treatment actually received based on clinical judgement.

\begin{tabular}{|c|c|c|c|c|c|}
\hline \multirow[b]{2}{*}{ Treatment } & \multicolumn{2}{|c|}{ Curative } & \multicolumn{2}{|c|}{ Palliative } & \multirow[b]{2}{*}{$p$-Value } \\
\hline & Unfit & Frail & Unfit & Frail & \\
\hline No. of patients & 17 & 18 & 11 & 48 & \\
\hline Median age & 78 & 78 & 83 & 82 & $0.001^{*}$ \\
\hline Ann Arbor stage III-IV & $69 \%$ & $78 \%$ & $45 \%$ & $49 \%$ & $0.02^{*}$ \\
\hline B symptoms & $33 \%$ & $45 \%$ & $10 \%$ & $32 \%$ & NS \\
\hline IPI risk class intermediate-high/high & 56 & 58 & 30 & 52 & NS \\
\hline $\mathrm{ORR}(\mathrm{CR}+\mathrm{PR})$ & $14(82 \%)$ & $13(72 \%)$ & $7(64 \%)$ & $25(52 \%)$ & NS \\
\hline Relapse rate & $2 / 14$ & $4 / 13$ & $2 / 7$ & $5 / 25$ & \\
\hline Lymphoma death & $60 \%$ & $80 \%$ & $80 \%$ & $84 \%$ & NS \\
\hline Non-hematologic toxicity $>2$ & $40 \%$ & $50 \%$ & $40 \%$ & $33 \%$ & NS \\
\hline
\end{tabular}

ORR, overall response rate; CR, complete remission; PR, partial remission; NS, not significant.

*Comparison between all patients who received curative vs. palliative treatment. 


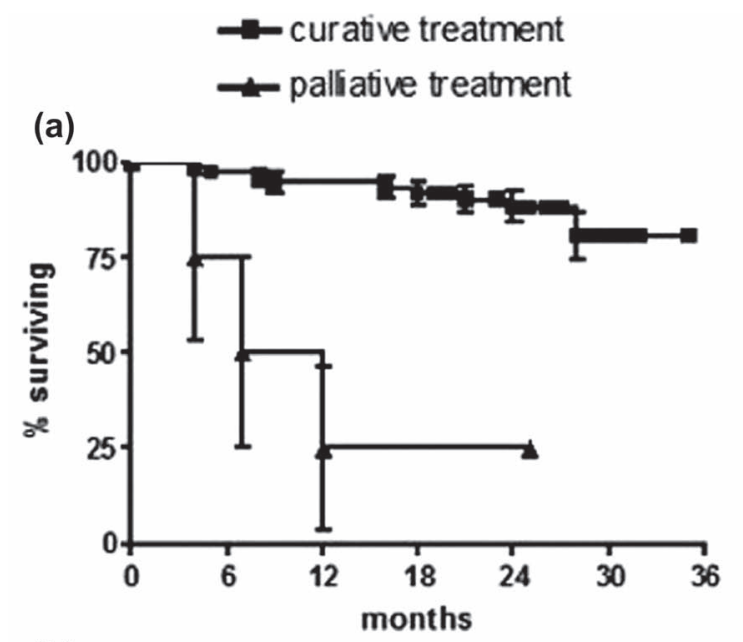

(b)

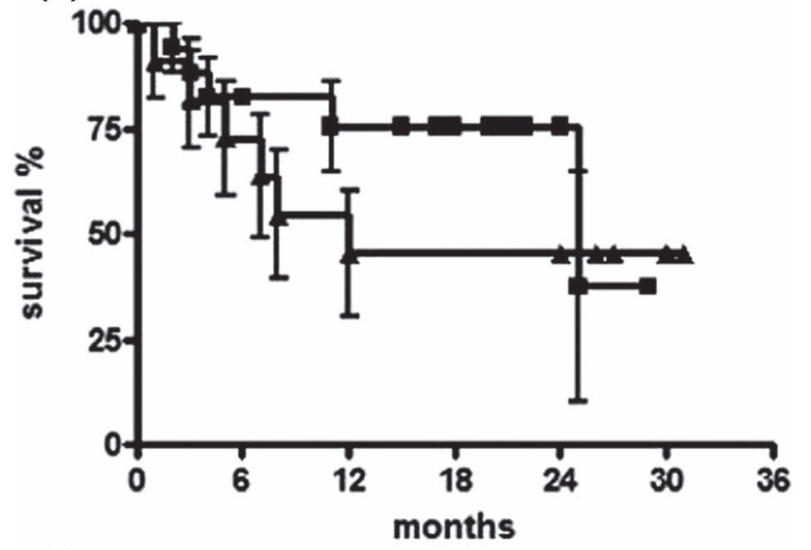

(c)

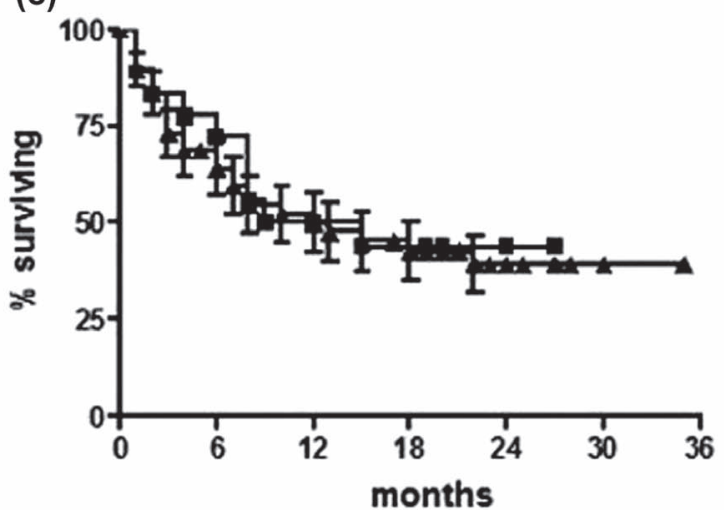

Figure 2. Actuarial overall survival curves of fit (a), unfit (b) and frail (c) elderly patients with DLBCL, each group subdivided according to treatment received (curative vs. palliative).

with OS. Within a multivariate analysis only IPI and CGA maintained their strong association with OS (Table IV).

\section{Discussion}

The results of our study confirm that CGA is a strong independent predictor of OS $[12,13]$ and can be usefully applied to the treatment decision in elderly patients with DLBCL.

We adopted age 70 as the lower limit of clinical senescence, since the prevalence of age-related changes is represented by almost a plateau up to this age and increases sharply with older age $[3,14]$.
We used a modified score originally proposed by Balducci and Extermann [3] that we validated in a small population of elderly patients with DLBCL [4] and that Spina et al. had already proposed as a modified version [8]. The primary aim was to try to further divide the category of frail patients in order to modulate treatment intensity. A fully standardized geriatric assessment tool is not yet available. Therefore, any choice in subdivision of the geriatric categories is somewhat arbitrary. However, the criteria we applied referred to validated rating scales and to previously published studies $[4,15]$.

Only short and rapid screening instruments can be widely accepted in oncology clinical practice $[16,17]$. This test is easy to perform, and does not require more than $15 \mathrm{~min}$. Trained clinicians and nurses can work together according to their skills, assigning the comorbidity and ADL/IADL scores, respectively. The most important caution is to avoid defining a patient as frail because of a reduced performance status depending only on his advanced and symptomatic disease. This may carry a significant risk of undertreating the patient.

More recently, great interest has been directed toward the prognostic role of nutritional parameters in the outcome of the geriatric population with cancer. Following observation of the close correlation between hypoalbuminemia and survival in elderly patients with DLBCL [18], sarcopenia was studied in this category of patients and was found to be a strong predictor of OS, but this approach is still not easily applicable in worldwide clinical practice [19].

The results of this study, far from being a final recommendation to be adopted in clinical practice, represent a further step toward the development of a simple geriatric score able to define different categories of elderly patients with DLBCL suitable for different treatment intensities.

According to the standard protocols for elderly patients with DLBCL [20], combination chemotherapy with CHOP or CHOP-like regimen with rituximab was chosen as the treatment strategy with curative intent. A relative dose intensity of more than $70 \%$ of the standard dose was considered acceptable to maintain its therapeutic effect, as already demonstrated [21].

Only patients classified as fit by CGA had a very satisfactory clinical outcome using the intensive curative approach. The 2-year OS rate of $88 \%$ did not differ from results achieved in younger patients with the same treatment [22,23]. Moreover, major toxic effects were limited, and only two patients died due to treatment-related toxicity. The benefit of curative versus palliative treatment was lost in non-fit patients, who had a poor outcome irrespective of the type of treatment received. The same results were obtained in a recent study by Marchesi et al., who used a similar simplified CGA [24]. In our study, frail patients more frequently had a worse IPI score and B symptoms compared with fit patients, although the difference was not significant; however, significantly better survival of fit compared to frail intensively treated patients was evident both in the subgroup of low and high IPI score, and in the subgroup of A and B stage, eliminating possible bias (data not shown). Compared to frail patients the outcome of unfit patients was not significantly different. However, there was a clear trend toward better survival, 
Table IV. Overall survival time according to patient and treatment characteristics (univariate and multivariate Cox regression analysis).

\begin{tabular}{|c|c|c|c|c|}
\hline Variables & $\begin{array}{c}\text { Univariate HR } \\
(95 \% \mathrm{CI})\end{array}$ & $p$-Value & $\begin{array}{c}\text { Multivariate HR } \\
(95 \% \mathrm{CI})\end{array}$ & $p$-Value \\
\hline Age $<80$ vs. $\geq 80$ years & $2.67(1.61-4.44)$ & 0.0002 & & \\
\hline Stage I-II vs. III-IV & $1.59(0.92-2.74)$ & 0.09 & & \\
\hline IPI (intermediate-low/low vs. intermediate-high/high) & $3.72(1.80-7.68)$ & 0.0003 & $4.60(1.35-15.64)$ & 0.008 \\
\hline CGA & $5.61(2.95-10.64)$ & 0.0001 & $3.69(1.09-12.51)$ & 0.03 \\
\hline $\mathrm{ADL}(\leq 5$ vs. 6$)$ & $0.3(0.17-0.51)$ & 0.0001 & & \\
\hline $\operatorname{IADL}(\leq 6$ vs. $\geq 7)$ & $0.24(0.14-0.41)$ & 0.0001 & & \\
\hline CIRS-G grade $2(<5$ vs. $\geq 5)$ & $2.89(1.04-8.03)$ & 0.04 & & \\
\hline CIRS-G grade $3-4(0$ vs. $\geq 1)$ & $2.14(1.22-3.73)$ & 0.007 & & \\
\hline Curative vs. palliative treatment approach & $0.27(0.16-0.46)$ & 0.0001 & & \\
\hline Treatment dose ( $<70 \%$ vs. $\geq 70 \%)$ & $0.38(0.17-0.86)$ & 0.02 & & \\
\hline
\end{tabular}

IPI, International Prognostic Index; CGA, comprehensive geriatric assessment; ADL, activity of daily living; IADL, instrumental activity of daily living; CIRS-G, Cumulative Illness Rating Score for Geriatrics; HR, hazard ratio; CI, confidence interval.

especially in the subgroup treated with curative intent. While the small number of unfit patients identified by CGA criteria may have limited the statistical power of the study, these results suggest that efforts in modulating the treatment intensity in this intermediate category of patients might be worthwhile in order to further improve their outcome, and to reduce the number of elderly patients who might benefit from palliative care only. Modulation of chemotherapy according to CGA has already been proposed by different authors $[8,13]$. Spina et al. obtained interesting results by adjusting the single chemotherapeutic agent according to comorbidities and total doses according to ADL/IADL. An attenuated immunochemotherapy regimen (rituximabminiCHOP) was used by a French group in elderly patients aged over 80 years without any other CGA parameter selection, obtaining a good compromise between efficacy and safety [18]. The combination of a lower dose of immunochemotherapy with a biological drug could also be a valid alternative to be tested in a clinical trial.

In conclusion, the CGA used in our study proved to be an efficient method to identify elderly patients with DLBCL who can benefit from a curative approach with anthracyclinecontaining immuno-chemotherapy. It further suggests the potential usefulness of identifying different risk groups in the category of non-fit patients, by showing that a proportion of unfit patients exist who could obtain significant benefit when treated with curative intent. Therefore, new risk-adapted strategies of treatment through modulated-intensity programs could reduce the difference of survival between fit and unfit patients, and clinical trials in this setting should be planned. Palliation seems the best choice for frail patients, and improvement of supportive care is the only effort that might be proposed in this category of patients.

Potential conflict of interest: Disclosure forms provided by the authors are available with the full text of this article at www.informahealthcare.com/lal.

\section{References}

[1] Extermann M, Aapro M, Bernabei R, et al. Use of comprehensive geriatric assessment in older cancer patients: recommendations from the task force on CGA of the International Society of Geriatric Oncology (SIOG). Crit Rev Oncol Hematol 2005;55:241-252.
[2] Fields PA, Linch DC. Treatment of the elderly patients with diffuse large B cell lymphoma. Br J Haematol 2012;157:159-170.

[3] Balducci L, Extermann M. Management of cancer in older person:a practical approach. Oncologist 2000;5:224-237.

[4] Tucci A, Ferrari S, Bottelli C, et al. A comprehensive geriatric assessment is more effective than clinical judgment to identify elderly diffuse large cell lymphoma patients who benefit from aggressive therapy. Cancer 2009;115:4547-4553.

[5] Miller MD, Towers A. A manual of guidelines for scoring the Cumulative Illness Rating Scale for Geriatrics (CIRSG). Pittsburg, PA: University of Pittsburg; 1991.

[6] Katz S, Akpom CA. A measure of primary sociobiological functions. Int J Health Serv 1976;6:493-508.

[7] Lawton MP, Brody EM. Assessment of older people: selfmantaining and instrumental activities of daily living. Gerontologist 1969;9:179-186.

[8] Spina M, Balzarotti M, Uziel L, et al. Modulated chemotherapy according to modified comprehensive geriatric assessment in 100 consecutive elderly patients with diffuse large B-cell lymphoma. Oncologist 2012;17:838-846.

[9] Kaplan EL, Meier P. Nonparametric estimation from incomplete observations. J Am Stat Assoc 1958;53:457-481.

[10] Mantel N. Evaluation of survival data and two new rank order statistics arising in its consideration. Cancer Chemother Rep 1966;50:163-170.

[11] Cox D. Regression models and life tables. J R Stat Soc B 1972; 34:187-202.

[12] Winkelmann N, Petersen I, Kiehntopf M, et al. Results of comprehensive geriatric assessment effect survival in patients with malignant lymphoma. J Cancer Res Clin Oncol 2011;137:733-738.

[13] Olivieri A, Gini G, Bocci C, et al. Tailored therapy in an unselected population of 91 elderly patients with DLBCL prospectively evaluated using a simplified CGA. Oncologist 2012;17:663-672.

[14] Balducci L, Schonwetter R, Gray G, et al. Individualized treatment of the older cancer patient: a decision analysis. J Am Geriatr Soc 1990;58:61a.

[15] Merli F, Luminari S, Rossi G, et al. Outcome of frail elderly patients with diffuse large B-cell lymphoma (DLBCL) prospectively identified by comprehensive geriatric assessment (CGA). Results from a study of the Fondazione Italiana Linfomi (FIL). Leuk Lymphoma 2014;55:38-43.

[16] Soubeyran P, Bellera CA, Gregoire F, et al. Validation of a screening test for elderly patients in oncology. J Clin Oncol 2008;26(15 Suppl.): Abstract 20568.

[17] Bellera CA, Rainfray M, Mathoulin-Pélissier S, et al. Screening older cancer patients: first evaluation of the G-8 geriatric screening tool. Ann Oncol 2012;23:2166-2172.

[18] Peyrade F, Jardin F, Thieblemont C, et al. Attenuated immunochemotherapy regimen (R-miniCHOP) in elderly patients older than 80 years with diffuse large B-cell lymphoma: a multicentre, single-arm, phase 2 trial. Lancet Oncol 2011;12:460-468.

[19] Lanic H, Tauzia JK, Modzelewski R, et al. Sarcopenia is an independent prognostic factor in elderly patients with diffuse large B-cell lymphoma treated with immunochemotherapy. Leuk Lymphoma 2014;55:817-823.

[20] Feugier P, Van Hoof A, Sebban C, et al. Long-term results of the R-CHOP study in the treatment of elderly patients with diffuse large B-cell lymphoma: a study by the Groupe d'Etude des Lymphomes de l'Adulte. J Clin Oncol 2005;23:4117-4126. 
[21] Hirakawa $\mathrm{T}$, Yamaguchi $\mathrm{H}$, Yokose $\mathrm{N}$, et al. Importance of maintaining the relative dose intensity of CHOP-like regimens combined with rituximab in patients with diffuse large B-cell lymphoma. Ann Hematol 2010;89:897-904.

[22] Pfreundschuh $\mathrm{M}$, Trümper L, Osterborg A, et al. CHOP-like chemotherapy plus rituximab versus CHOP-like chemotherapy alone in young patients with good-prognosis diffuse large-B-cell lymphoma: a randomised controlled trial by the MabThera International Trial (MInT) Group. Lancet Oncol 2006;7:379-391.

\section{Supplementary material available online}

Supplementary Figure showing subdivision of patients
[23] Schmitz N, Nickelsen M, Ziepert M, et al. Conventional chemotherapy (CHOEP-14) with rituximab or high-dose chemotherapy (MegaCHOEP) with rituximab for young, high-risk patients with aggressive B-cell lymphoma: an open-label, randomised, phase 3 trial (DSHNHL 2002-1). Lancet Oncol 2012;13:1250-1259.

[24] Marchesi F, Cenfra N, Altomare L, et al. A retrospective study on 73 elderly patients ( $\geq 75$ years) with aggressive B-cell non Hodgkin lymphoma: clinical significance of treatment intensity and comprehensive geriatric assessment. J Geriatr Oncol 2013;4:242-248. 